\title{
Mucormicosis pulmonar en niños. Presentación de 3 casos y revisión de la literatura
}

\author{
Víctor Manuel Carrillo-Rodríguez,凶Mario Soto-Ramos, Raúl Hernández-Saldaña, \\ Luis Carlos Hinojos-Gallardo, Salvador González-Ortiz
}

Servicio de Neumología Pediátrica y Cirugía de Tórax, Hospital Infantil de Especialidades de Chihuahua, México. Trabajo recibido: 19-VIII-2016; aceptado: 09-III-2017

\begin{abstract}
RESUMEN. La mucormicosis es una infección grave causada por hongos del género Mucor y Rizhopus (del orden Mucorales, clase: Zygomycetes) que se presenta en pacientes inmunocomprometidos, constituye una micosis oportunista. Ingresan al organismo por inhalación de las esporas a través de la vía aérea, contaminación de heridas o catéteres o por ingestión de las mismas. Las formas de presentación clínica son muy variadas; sin embargo, las más importantes son la rinocerebral y la pulmonar, siendo la última la más reportada en niños. El diagnóstico es difícil por lo que es necesario el hallazgo de las hifas en muestras de lavado broncoalveolar, secreciones corporales o biopsias. El tratamiento se basa en tres aspectos fundamentales: 1) tratamiento de la enfermedad de base, 2) tratamiento antimicótico y 3) debridación de tejido necrótico.
\end{abstract}

Palabras clave: Mucormicosis pulmonar, niños, micosis.

ABSTRACT. Mucormycosis is a serious infection caused by fungi of the genus Mucor and Rizhopus (order Mucorales, class: Zygomycetes) that occurs in immunocompromised hosts, so it is an opportunistic fungal infection. The enter way is the aspiration from contaminated soil with spores, contamination of wounds or catheters, or by ingestion. The most important clinical presentations are rhinocerebral and lung diseases; the latter being the most reported in children. Diagnosis is difficult, finding hyphae in bronchoalveolar lavage samples, body fluids or biopsies is necessary. The treatment is based on three fundamental aspects: 1) treatment of the underlying disease, 2) antifungal therapy and 3) debridement of necrotic tissue.

Key words: Pulmonary mucormicosis, children, micosis.

\section{INTRODUCCIÓN}

La mucormicosis es una enfermedad infecciosa ocasionada por hongos oportunistas omnipresentes en la naturaleza, de la clase de Zigomycetes, orden de los Mucorales de los cuales los géneros que habitualmente infectan al hombre son Rizhopus, Absidia y Mucor . $^{1,2}$ Corresponde al $1.5 \%$ de las infecciones fúngicas invasivas y su incidencia ha ido aumentando en el transcurso de los años. En Francia se reporta un aumento de 0.7 casos por millón de habitantes en 1997 a 1.2 casos por millón de habitantes en el 2006. ${ }^{1}$ En México, los casos son esporádicos y se diagnostican habitualmente en hospitales de tercer nivel. El Instituto Nacional de Pediatría reportó durante el período 1998-2010 una incidencia de 4\% de todas las micosis en las vías respiratorias. ${ }^{3}$ Mientras que el Hospital General de México reportó, en un estudio retrospectivo de 1985 al 2012, 168 casos de mucormicosis donde el $13.9 \%$ (22 casos) correspondían a niños. ${ }^{4}$

La mucormicosis está asociada a una condición subyacente, particularmente pacientes inmunocomprometidos. Los principales factores de riesgo son las neoplasias hematológicas (principalmente leucemia), neutropenia, diabetes mellitus (DM) descontrolada, trasplante de células hematopoyéticas u órgano sólido, inmunodeficiencias, quemaduras extensas, desnutrición severa e insuficiencia renal crónica. ${ }^{1,4} \mathrm{La}$ infección se adquiere principalmente a través de la inhalación de esporas, pero también está descrita la infección por ingestión de las mismas o contaminación de heridas o catéteres endovenosos. Las principales formas de presentación clínica son la rinocerebral (55\%), pulmonar (30\%), cutánea, gastrointestinal y diseminada. ${ }^{4,5}$ En niños, la forma rinocerebral es la más 
frecuente $(77 \%)$, mientras que la pulmonar se reporta en el $4 \%$ de los casos de mucormicosis. ${ }^{3,4,6}$

El cuadro clínico es inespecífico y tanto la presentación clínica como la radiológica es indistinguible de la aspergilosis pulmonar. Sin embargo, la afección pulmonar suele manifestarse por fiebre persistente asociado a síntomas pulmonares en un paciente inmunocomprometido a pesar del tratamiento antibiótico. La frecuencia de los síntomas es variable y los más observados con: fiebre (38-100\%), tos (19-92\%), disnea (19-81\%), dolor torácico (13-81\%) hemoptisis (13$31 \%))^{1,7,8}$ En la forma rinocerebral se pueden observar lesiones necróticas en cara hasta el $80 \%$ de los casos (escaras centinela). ${ }^{3}$

Los métodos de referencia para el diagnóstico de mucormicosis son el examen microscópico directo, el cultivo y el estudio histopatológico., ${ }^{1,8,9}$

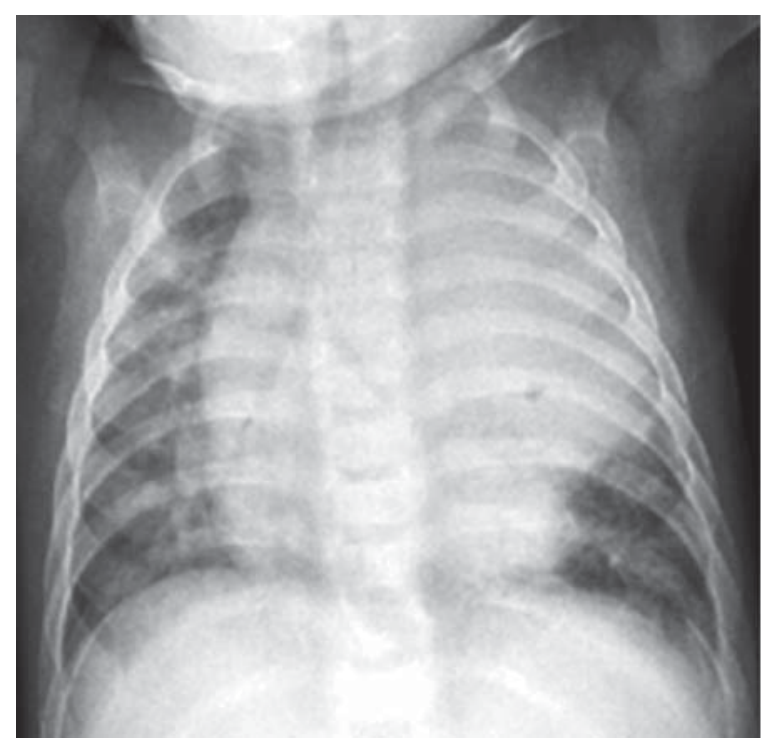

Figura 1. Radiografía AP de tórax.

\section{PRESENTACIÓN DE CASOS}

\section{Caso 1}

Paciente masculino de 5 meses, padre con tabaquismo positivo, abuelos paternos con DM e HTA. Nace por parto eutócico a término, peso de $2,700 \mathrm{~g}$, talla $49 \mathrm{~cm}$. APGAR 8/9. Lactancia materna por dos meses, sin ablactación. Estado de vacunación incompleto (sólo hepatitis B y BCG al nacimiento). Síntomas respiratorios desde el primer mes de vida con tos productiva, sin predominio de horario, asociado a constipación nasal y rinorrea hialina en forma episódica. Hospitalizado a los dos meses de vida durante cuatro días por bronquiolitis, más tarde dos visitas a urgencias por dificultad respiratoria tratado con salbutamol inhalado y antibiótico. Segunda hospitalización a los cuatro meses por neumonía del lóbulo superior izquierdo durante siete días recibiendo tratamiento con cefuroxima. Se refieren dos episodios de candidiasis oral tratada con nistatina. Cuadro clínico de tres semanas de evolución con fiebre de $39^{\circ} \mathrm{C}$ de predominio nocturno, posteriormente con tos productiva en accesos cortos, sin predominio de horario, acompañado de rinorrea hialina. Un día previo a su hospitalización inicia con aumento del esfuerzo respiratorio y tos cianosante.

A la exploración física con mucosa nasal y faríngea eritematosa, sin exudado, ni hipertrofia amigdalina, cuello sin adenopatías, tórax con movimientos respiratorios asimétricos (disminuidos en hemitórax izquierdo), ruidos respiratorios disminuidos en hemitórax izquierdo, con aumento de las vibraciones vocales y matidez a la percusión en región apical izquierda. Crépitos gruesos diseminados, sin sibilancias. Ruidos cardíacos normales. Abdomen sin hepatomegalia, peristalsis normal, pulsos distales normales.

Exámenes de laboratorio. BHC: $\mathrm{Hb} 9.7 \mathrm{~g} / \mathrm{dL}$, Hto $30 \%$, leucocitos $20,200 \mathrm{cel} / \mathrm{mm} 3$, neutrófilos $59 \%$,
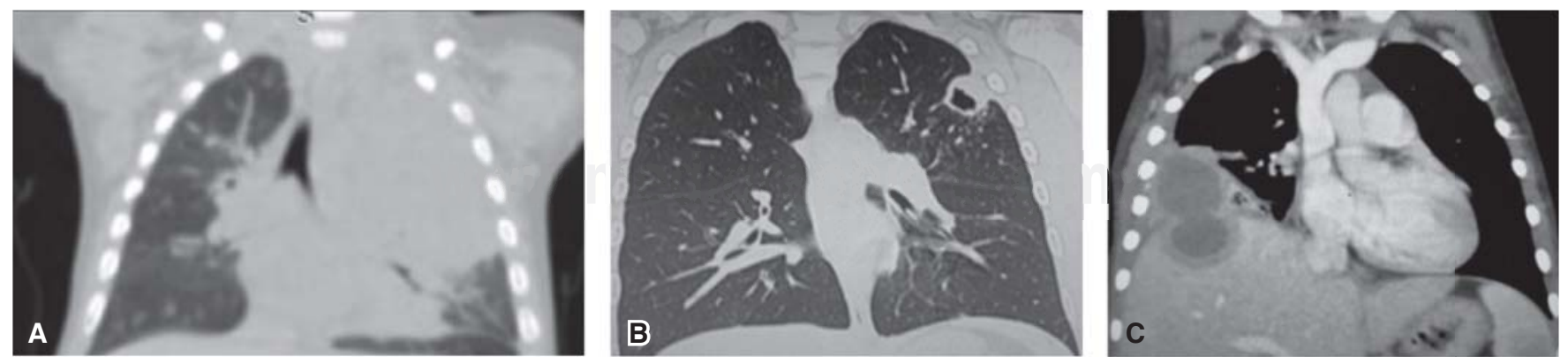

Figura 2. Hallazgos tomográficos en cortes coronales. A) Masa pulmonar en lóbulo superior izquierdo con desplazamiento y compresión de estructuras mediastinales. B) Lesión cavitada del lóbulo superior izquierdo en un paciente con hemoptisis. C) Absceso hepático asociado a derrame pleural derecho. 
linfocitos $34 \%$, monocitos $6 \%$, plaquetas $358,000 \mathrm{cel} /$ $\mathrm{mm}^{3}$. PCR $=1: 320, \mathrm{VSG}=20 \mathrm{mmHr}$, proteínas totales en $4 \mathrm{~g} / \mathrm{dL}$, albúmina $2.5 \mathrm{~g} / \mathrm{dL}$, DHL $560 \mathrm{U} / \mathrm{L}$.

Radiografía de tórax AP (figura 1) con opacidad homogénea en lóbulo superior izquierdo que desplaza las estructuras mediastinales al lado derecho, sin broncograma. La tomografía de tórax (figura 2-A) muestra una masa pulmonar con infiltración de estructuras mediastinales.

Se realizó biopsia a cielo abierto de la lesión tumoral ante sospecha de linfoma; aunque el reporte histopatológico determinó la presencia de reacción granulomatosa crónica (figura 3) con trombosis vascular y presencia de múltiples hifas de Mucor en zonas perivasculares y granulomas.

\section{Caso 2}

Paciente femenino de 12 años sin antecedentes heredofamiliares de importancia. En la vivienda existe abundante humedad con moho en las paredes de las habitaciones. Presentó cuadro de varicela a los cuatro años de edad, sin complicaciones. A esa misma edad también manifestó cuadro de sibilancias tratado con salbutamol por dos semanas con mejoría de los síntomas. Rinitis alérgica desde los cuatro años de vida en tratamiento con esteroide nasal y montelukast.

Cuadro clínico de cuatro días de evolución con tos productiva al inicio esporádica y después en accesos cortos, sin cianosis o vómitos posteriores a los accesos de tos, no existió predominio de horario y no refirió factores exacerbantes ni atenuantes de la tos. Más tarde inició con hemoptoicos, de cantidad escasa

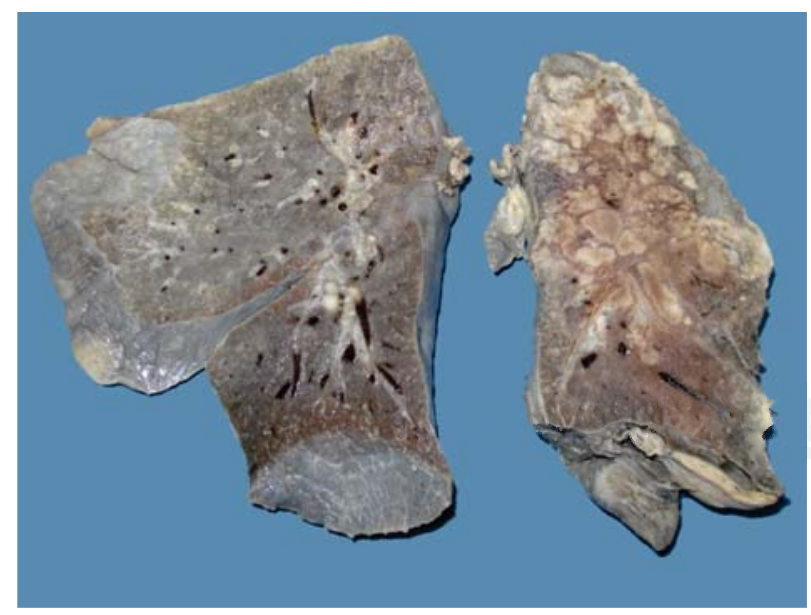

Figura 3. Pieza de necropsia correspondiente a bloque pulmonar del caso 1. Se observan múltiples granulomas con necrosis central que predominan en lóbulo superior izquierdo. (estrías de sangre en el esputo) en una ocasión por día. Negó la presencia de fiebre, diaforesis, pérdida de peso, síntomas nasales, disnea o dificultad respiratoria. Un día previo a su hospitalización con aumento de la expectoración hemoptoica (esputo rojo) de escasa cantidad, en número de cuatro ocasiones, así como disnea de pequeños esfuerzos.

A la exploración física: con mucosa nasal pálida, con hipertrofia de cornetes, sin huellas de sangrado. Inyección conjuntival bilateral. Otoscopia normal. Faringe levemente hiperémica, sin hipertrofia amigdalina ni exudado. Cuello sin adenopatías. Tórax con movimientos respiratorios simétricos, ruidos respiratorios normales, sin fenómenos agregados, claro pulmonar a la percusión. Ruidos cardíacos normales. Abdomen asignológico. Pulsos distales normales.

Exámenes de laboratorio: $\mathrm{Hb} 14.2 \mathrm{~g} / \mathrm{dL}$, Hto 43\%, leucocitos $10,200 \mathrm{cel} / \mathrm{mm}^{3}$, neutrófilos $50 \%$, linfocitos $47 \%$, eosinófilos $1 \%$, monocitos $2 \%$, plaquetas 305,000 $\mathrm{cel} / \mathrm{mm}^{3}$. PCR negativa, VSG $10 \mathrm{mmHr}$. En la radiografía PA de tórax se observó una lesión radiolúcida, oval, con pared delgada y bordes regulares, sin presencia de nivel hidroaéreo y sin desplazamiento de estructuras adyacentes, localizada en el lóbulo superior izquierdo. Se realizó TC de tórax mostrando lesión cavitaria de $1.4 \mathrm{~mm}$ en lóbulo superior izquierdo, sin lesiones a otro nivel ni alteración mediastinal (figura 2-B).

Ante sospecha de tuberculosis pulmonar se realizó PPD reportándose en $7 \mathrm{~mm}$ baciloscopias negativas y GENEXPERT MTB negativo en LBA. Inmunoglobulinas séricas normales, $p$-ANCA y $\mathrm{C}$-anca negativos. Debido a persistencia de la hemoptisis se realizó resección quirúrgica de la lesión cavitaria. El reporte histopatológico determinó la presencia de múltiples hifas de Mucor perivasculares. Tratada con itraconazol por cuatro meses con evolución clínica favorable.

\section{Caso 3}

Paciente masculino de 8 años sin antecedentes heredofamiliares, perinatales o personales patológicos de importancia para el padecimiento actual. Recién diagnóstico de leucemia linfoblástica aguda Pre-B con cromosoma Philadelphia, $t(9 ; 22)(q 34 ; q 11)$, en tratamiento con vincristina y daunorrubicina. Siete días previos a su hospitalización inició con fiebre no cuantificada, rinorrea hialina y aumento de volumen en región submaxilar derecha. Tratado con azitromicina e ibuprofeno sin mejoría. Posteriormente con tos productiva y dolor en hombro derecho.

A la exploración con mucosa nasal y faríngea con eritema leve, sin hipertrofia amigdalina, otoscopia normal. Cuello con adenopatía en cadena cervical pos- 
terior derecha de $1 \mathrm{~cm}$ de diámetro, móvil y dolorosa a la palpación. Tórax con movimientos respiratorios simétricos, ruidos respiratorios con disminución del murmullo vesicular en región basal derecha y presencia de estertores crepitantes bilaterales. La percusión del tórax se encontraba con claro pulmonar y disminución de las vibraciones vocales en la región basal derecha a la palpación. Abdomen con dolor mal definido a la palpación profunda en hipocondrio derecho, sin hepatomegalia, sin datos de irritación peritoneal y la peristalsis adecuada. El resto de la exploración sin hallazgos relevantes.

Exámenes de laboratorio con HB $6.0 \mathrm{~g} / \mathrm{dL}$, Hto $16.3 \%$, leucocitos $3,100 \mathrm{cel} / \mathrm{mm}^{3}$, neutrófilos absolutos en $1,000 \mathrm{cel} / \mathrm{mm}^{3}$, plaquetas $70,000 \mathrm{cel} / \mathrm{mm}^{3}$, TP 13 s, TPT 53s, DHL 632 UI/I, AST 35 UI/L, ALT $18 \mathrm{UI} / \mathrm{L}$, albúmina $3.7 \mathrm{~g} / \mathrm{dL}$; el resto de los exámenes dentro de parámetros normales. Se tomó radiografía de tórax con presencia de derrame pleural derecho, pequeño y consolidación del lóbulo medio.

Se inició tratamiento con vancomicina, meropenem y fluconazol. Después manifestó incremento de intensidad del dolor abdominal, predominando en el cuadrante superior derecho de tipo punzante, acompañado de disnea de pequeños esfuerzos, dolor en el hombro derecho y tos no productiva en accesos cortos, sin producir cianosis o vómito y que exacerbaba el dolor abdominal. Ante ello se decidió realizar TAC de tórax contrastada, la cual muestra un absceso hepático que se comunica con la cavidad pleural a través del diafragma (figura 2-C). Se realiza biopsia percutánea guiada por TAC del absceso hepático para estudio microbiológico; empero, fue necesario el aseo quirúrgico de las cavidades (pleural y del absceso hepático). El reporte histopatológico muestra la presencia de múltiples hifas no septadas correspondientes a Mucor en tejido hepático (figura 4-A). El paciente continuó en tratamiento con itraconazol por cuatro semanas con mejoría clínica y radiográfica posterior.

\section{DISCUSIÓN}

El diagnóstico de mucormicosis pulmonar es difícil y el cuadro clínico/radiológico es indistinguible de la aspergilosis pulmonar invasiva, por lo que todos los pacientes con cuadro clínico sugestivo de infección fúngica invasiva asociado a factores de riesgo deben realizarse tomografía de alta resolución de tórax. ${ }^{10}$

No existe patrón radiográfico o tomográfico patognomónico de mucormicosis pulmonar; sin embargo, existen los siguientes patrones: nódulos solitarios $(1-3 \mathrm{~cm})$ en el $41-80 \%$ de los casos, nódulos múltiples (> 10) en 31-64\%, masa $(>3 \mathrm{~cm}$ ) en $31-88 \%$,
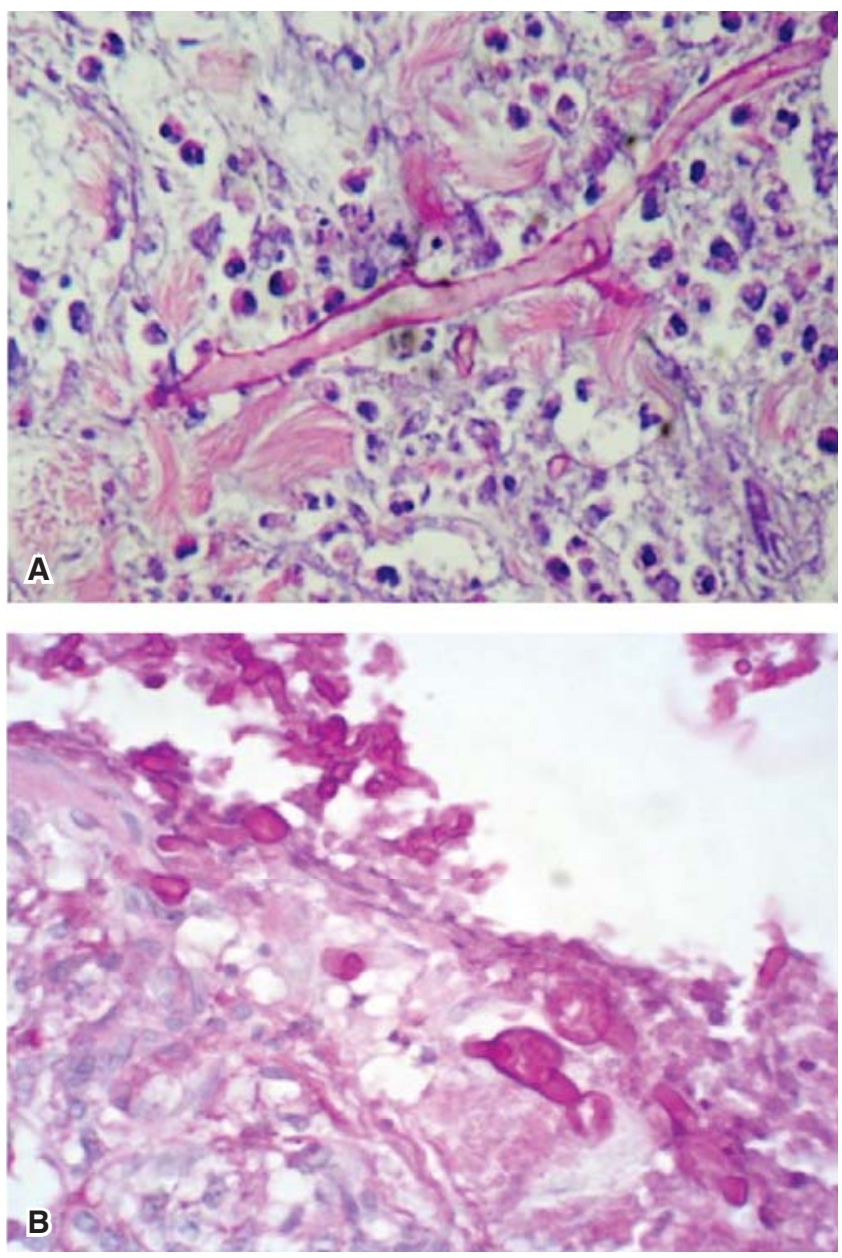

Figura 4. Estudio histopatológico de casos 1 y 3. A) Biopsia de absceso hepático con derrame pleural. Se observa hifa aceptada de $25 \mu \mathrm{m}$ con ramificaciones en ángulos rectos. B) Biopsia de tejido pulmonar observándose hifas de Mucor en cortes longitudinales y transversales.

micronódulos en $6-55 \%$, signo del «Halo» en $25-32 \%$, signo del «Halo inverso» en 4-94\%, cavitaciones en > $37 \%$, derrame pleural en $12-63 \% .{ }^{11,12}$ Las alteraciones tomográficas observadas en nuestros pacientes corresponden a una neumonía organizada con múltiples granulomas en el lóbulo superior izquierdo (figura 2-A). El segundo paciente presentó una cavitación en lóbulo superior izquierdo (figura 2-B) y el tercer paciente desarrolló derrame pleural asociado a absceso hepático (figura 2-C).

A diferencia de la aspergilosis, en los Mucorales no existen $\beta$-D-glucanos en la pared celular por lo que no se detectan galactomananos ni $\beta$-D-glucanos en la mucormicosis. ${ }^{13}$ Los métodos recomendados para el diagnóstico de mucormicosis continúan siendo la examinación microscópica directa, el cultivo y el 
estudio histopatológico, por lo tanto, se recomienda la obtención de muestras de secreciones o tejido. ${ }^{1} \mathrm{El}$ lavado broncoalveolar (LBA) se realiza en los pacientes con lesiones pulmonares y cuando éste no es posible se recomienda la biopsia percutánea guiada por TC. Cuando las lesiones pulmonares no son accesibles para abordaje percutáneo debe realizarse biopsia pulmonar quirúrgica. ${ }^{14} \mathrm{El}$ estudio histopatológico muestra la presencia de hifas no septadas con esporangios de hasta $300 \mu \mathrm{m}$, con ramificaciones en ángulos rectos acompañada de necrosis y angioinvasión ${ }^{1}$ (figura 4-B). En nuestros casos sólo en el primero se realizó LBA, pero se reportó negativo para hongos, el diagnóstico definitivo fue en el estudio histopatológico de la pieza quirúrgica; lo mismo sucedió con el caso 2 donde se reseca la lesión cavitaria. En el caso 3 se realizó el diagnóstico mediante biopsia pulmonar percutánea guiada por TC debido a que la lesión era periférica y accesible. Hoy se sabe que el ADN de los Mucorales puede ser detectado hasta dos meses antes de la aparición de lesiones pulmonares, ${ }^{1}$ por lo que se están desarrollando métodos diagnósticos mediante reacción en cadena de la polimerasa (PCR) para el diagnóstico de infecciones fúngicas invasivas. ${ }^{15}$

El tratamiento de la mucormicosis pulmonar depende de tres aspectos fundamentales: 1) el control de la enfermedad de base, 2) el tratamiento antifúngico, y 3) la debridación quirúrgica del tejido necrótico (cuando sea posible). Cuanto más factores de riesgo existan el tratamiento será más difícil y mayor la mortalidad, a pesar de un tratamiento antifúngico adecuado. ${ }^{16} \mathrm{El}$ tratamiento antifúngico debe instaurarse tan pronto como se establezca el diagnóstico. Se recomienda el inicio del tratamiento con anfotericina liposomal o complejo lipídico, ya que tiene excelente actividad contra Mucorales con dosis de hasta $5 \mathrm{mg} / \mathrm{kg}$ por día. El posaconazol también muestra actividad contra Mucorales, con el beneficio de que se puede administrar vía oral y con pocos efectos secundarios. El Itraconazol muestra actividad limitada en algunos casos, mientras que el vorizonazol, fluconazol y la caspofungina tiene escasa actividad frente a Mucorales. ${ }^{17}$ La duración del tratamiento depende del grado de afección y de la enfermedad subyacente, pero en general puede ser hasta 6-8 semanas. ${ }^{18} \mathrm{La}$ combinación de tratamiento médico con quirúrgico disminuye la mortalidad de un 68 a un $11 \%$ cuando se compara con tratamiento médico únicamente. ${ }^{1,19}$ La debridación de tejido necrótico debe ser agresiva, para permitir un mejor control de la infección. ${ }^{19}$

La mucormicosis pulmonar tiene alta mortalidad, reportada en la literatura hasta en un $54 \%$ en las series de casos más grandes, aunque ésta depende del sitio de la infección, siendo las formas diseminada y pulmonar las de mayor mortalidad; así como de la presencia de factores de riesgo y uso temprano de antifúngicos. ${ }^{20}$ De los tres pacientes que presentamos, sólo el paciente del caso 1 falleció, siendo éste el único caso en donde no se realizó debridación de tejido necrótico. Los otros recibieron tratamiento con itraconazol y cirugía con debridación de tejido necrótico presentando recuperación total.

\section{CONCLUSIÓN}

La mucormicosis pulmonar es una entidad rara en niños cuyo diagnóstico se establece mediante estudio microscópico directo, cultivo o histopatología de los tejidos o secreciones. Si no existe un control adecuado de los factores de riesgo o la debridación de tejido necrótico, la mortalidad es alta a pesar de un tratamiento antifúngico adecuado.

\section{REFERENCIAS}

1. Danion F, Aguilar C, Catherinot E, et al. Mucormycosis: New developments into a persistently devastating infection. Semin Respir Crit Care Med 2015;36(5):692705. doi: 10.1055/s-0035-1562896.

2. Chayakulkeeree M, Ghannoum MA, Perfect JR. Zygomycosis: the reemerging fungal infection. Eur J Clin Microbiol Infect Dis 2006;25(4):215-229.

3. Vázquez TO, Campos RT, Ovando FJE. Micosis en vías respiratorias en niños. Rev Enfer Infec Pediatr 2011;XXIV(95):88-89.

4. Bonifaz A, Tirado-Sánchez A, Calderón L, et al. Mucormycosis in children: a study of 22 cases in a Mexican hospital. Mycoses 2014;57 Suppl 3:79-84. doi: 10.1111/myc.12233.

5. Munguía CDA, Ochoa GCC, Peña MES, Romo GJ, Téllez BJL. Mucormicosis pulmonar. Presentación de un caso. Neumol Cir Torax 2009;68(2):78-81.

6. Skiada A, Pagano L, Groll A, et al.; European Confederation of Medical Mycology Working Group on Zygomycosis. Zygomycosis in Europe: analysis of 230 cases accrued by the registry of the European Confederation of Medical Mycology (ECMM) Working Group on Zygomycosis between 2005 and 2007. Clin Microbiol Infect 2011;17(12):1859-1867. doi: 10.1111/j.1469-0691.2010.03456.x.

7. Chakrabarti A, Chatterjee SS, Das A, et al. Invasive zygomycosis in India: experience in a tertiary care hospital. Postgrad Med J 2009;85(1009):573-581. doi: 10.1136/pgmj.2008.076463.

8. Pagano L, Offidani M, Fianchi L, et al.; GIMEMA (Gruppo Italiano Malattie EMatologiche dell'Adulto) Infection Program. Mucormycosis in hematologic patients. Haematologica 2004;89(2):207-214. 
9. Garcia-Hermoso D, Alanio A, Lortholary O, Dromer F. Agents of systemic and subcutaneous mucormycosis and entomophthoromycosis. In: Manual Clinical Microbiology. 11th ed. Washington, DC: ASM Press; 2015.p.2087-2108.

10. Chamilos G, Marom EM, Lewis RE, Lionakis MS, Kontoyiannis DP. Predictors of pulmonary zygomycosis versus invasive pulmonary aspergillosis in patients with cancer. Clin Infect Dis 2005;41(1):60-66.

11. Vogl TJ, Hinrichs T, Jacobi V, Böhme A, Hoelzer D. Computed tomographic appearance of pulmonary mucormycosis. Rofo 2000;172(7):604-608.

12. Wahba H, Truong MT, Lei X, Kontoyiannis DP, Marom EM. Reversed halo sign in invasive pulmonary fungal infections. Clin Infect Dis 2008;46(11):1733-1737. doi: 10.1086/587991.

13. Marty FM, Koo S. Role of (1-->3)-beta-D-glucan in the diagnosis of invasive aspergillosis. Med Mycol 2009;47 Suppl 1:S233-S240. doi: 10.1080/13693780802308454.

14. Lass-Flörl C, Resch G, Nachbaur D, et al. The value of computed tomography-guided percutaneous lung biopsy for diagnosis of invasive fungal infection in immunocompromised patients. Clin Infect Dis 2007;45(7):e101-e104.

15. Millon L, Larosa F, Lepiller Q, et al. Quantitative polymerase chain reaction detection of circulating DNA in serum for early diagnosis of mucormycosis in immunocompromised patients. Clin Infect Dis 2013;56(10):e95-e101. doi: 10.1093/cid/cit094.

16. Saoulidis S, Simitsopoulou M, Dalakiouridou M, et al. Antifungal activity of posaconazole and granulocyte colony-stimulating factor in the treatment of disseminated zygomycosis (mucormycosis) in a neutropaenic murine model. Mycoses 2011;54(5):e486-e492. doi: 10.1111/j.1439-0507.2010.01958.x.

17. Almyroudis NG, Sutton DA, Fothergill AW, Rinaldi MG, Kusne S. In vitro susceptibilities of 217 clinical isolates of zygomycetes to conventional and new antifungal agents. Antimicrob Agents Chemother 2007;51(7):25872590.

18. Skiada A, Lanternier F, Groll AH, et al.; European Conference on Infections in Leukemia. Diagnosis and treatment of mucormycosis in patients with hematological malignancies: guidelines from the 3rd European Conference on Infections in Leukemia (ECIL 3). Haematologica 2013;98(4):492-504 doi: 10.3324/ haematol.2012.065110.

19. Tedder M, Spratt JA, Anstadt MP, Hegde SS, Tedder SD, Lowe JE. Pulmonary mucormycosis: results of medical and surgical therapy. Ann Thorac Surg 1994;57(4):10441050.

20. Roden MM, Zaoutis TE, Buchanan WL, et al. Epidemiology and outcome of zygomycosis: a review of 929 reported cases. Clin Infect Dis 2005;41(5):634-653.

\section{$\triangle$ Correspondencia:}

Dr. Víctor Manuel Carrillo-Rodríguez, Hospital Infantil de Especialidades del Estado de Chihuahua. Av. Pacheco s/n, colonia Ávalos, 31090, Ciudad Chihuahua, Chihuahua, México.

Correo electrónico: victormcarrillo2012@gmail.com

Los autores declaran no tener conflicto de intereses. 